\title{
A Monte Carlo-based Fiber Tracking Algorithm using Diffusion Tensor MRI
}

\author{
F.Prados, A.Bardera, M.Sbert, I.Boada and M.Feixas \\ Institut d'Informàtica i Aplicacions \\ Universitat de Girona \\ \{ferran.prados,anton.bardera,mateu.sbert,imma.boada,miquel.feixas\}@udg.es
}

\begin{abstract}
Diffusion tensor magnetic resonance imaging, which measures directional information of water diffusion in the brain, has emerged as a powerful tool for human brain studies. In this paper, we introduce a new Monte Carlo-based fiber tracking approach to estimate brain connectivity. One of the main characteristics of this approach is that all parameters of the algorithm are automatically determined at each point using the entropy of the eigenvalues of the diffusion tensor. Experimental results show the good performance of the proposed approach.
\end{abstract}

\section{Introduction}

Diffusion tensor magnetic resonance imaging (DT-MRI) has became a powerful tool in the investigation of the human brain. It provides in vivo and in a non-invasive way directional information of water diffusion in the brain white matter. Usually, this information is represented by a diffusion tensor, i.e., a symmetric second order tensor that represents the diffusion of water molecules in a voxel. From these data, tractography algorithms obtain explicit information of how fibers have to be connected.

Most of current tractography algorithms use the major diffusion tensor eigenvector to define the local fiber direction, reducing the diffusion information to one single main direction per voxel [4]. The main drawback of this approach arises when the tensor has not a strong directional component. Also, DT-MRI data noise [9] and partial volume effects [1] make difficult the computation of the fiber direction. To overcome all these limitations, more sophisticated tractography techniques have been proposed; amongst them, techniques based on high angular resolution acquisitions [14], regularization [3], tensor deflection [10] and stochasticity.

Stochastic fiber tracking techniques $[3,6,8,13]$ overcome the shortcomings of deterministic methods by adding some randomness to the deterministic tractography. They simulate a macroscopic random walk of a particle through the set of voxels. This random walk is defined by one-toone jumps where the step of the jump and its direction is determined by the parameters of the algorithm. After a finite number of iterations, a probability value could be computed and assigned to the tracts.

In this paper, we propose a new Monte Carlo approach to estimate the fiber tracks. This method is based on the introduction of the entropy of the diffusion tensor to obtain the track direction and the step size. Our proposal is evaluated by means of a brain dataset and is compared with other stochastic fiber tracking algorithms. This paper is organized as follows. In Section 2 we survey background and related work, and in Section 3 we present our method. Experimental results are given in Section 4.

\section{Background and Related Work}

In this section we review some basic diffusion tensor concepts and recent stochastic tractography approaches.

\subsection{Diffusion Tensor}

The diffusion tensor $D(x)$ is a symmetric second order tensor whose coefficients describe the diffusion of water molecules in a voxel $x$. Diagonalization of $D(x)$ provides three eigenvalues $\left(\lambda_{1}, \lambda_{2}, \lambda_{3}\right)$ and three eigenvectors $\left(e_{1}, e_{2}, e_{3}\right)$ that define the directions of main, medium and minimum diffusivity, respectively. Since $D(x)$ is symmetric, $\lambda_{i}$ are positive real numbers and the three eigenvectors $e_{i}$ are orthogonal and form a basis. Geometrically, $D(x)$ can be thought as an ellipsoid with its three axes oriented along the eigenvectors and with the three semi-axis lengths proportional to the square root of the eigenvalues of the tensor-mean diffusion distances [2]. Using this ellipsoidal interpretation, the diffusion properties of a tissue can be classified according to the shape of the ellipsoids: extended ellipsoids correspond to regions with strong linear diffusion, flat ellipsoids to planar diffusion and spherical ellipsoids to 
regions of isotropic media. Also, a quantitative classification can be done through the coefficients $c_{l}=\frac{\lambda_{1}-\lambda_{2}}{\lambda_{1}+\lambda_{2}+\lambda_{3}}$, $c_{p}=\frac{2\left(\lambda_{2}-\lambda_{3}\right)}{\lambda_{1}+\lambda_{2}+\lambda_{3}}$ and $c_{s}=\frac{3 \lambda_{3}}{\lambda_{1}+\lambda_{2}+\lambda_{3}}$ corresponding to linear, planar and spherical diffusion [16]. These coefficients are normalized to range [0..1]. Values of $c_{l}$ that are close to 1 are regions with strong linear diffusion. Large $c_{p}$ values correspond to planar diffusion and large $c_{s}$ values to isotropic media. Large $c_{p}$ are markers of ambiguity tracking situations since they appear when more than a fiber is in the voxel because of crossing or kissing fibers, or branching or merging fibers.

\subsection{Stochastic fiber tracking}

A fiber path can be described as a sequence of points $x_{n} \in V$, where $V$ is a volume in $R^{3}$. Tracking algorithms determine how to obtain the sequence of points $x_{n}$ being $x_{0}$ the starting point for tracks. The principal eigenvector of tensor $D(x)$ is denoted by $e\left(D\left(x_{i}\right)\right)$. A normalized vector $\frac{b}{\|b\|}$ is denoted by $\bar{b}$.

Deterministic approaches obtain $x_{n}$ values following the maximum diffusion direction. Thus, if the direction of the starting point is $v_{0}=e\left(D\left(x_{0}\right)\right)$, then $x_{n}=x_{n-1}+\overline{v_{n-1}} \mu$, where $v_{n}=e\left(D\left(x_{n}\right)\right)$ and $\mu$ is the step size.

Stochastic fiber tracking techniques introduce randomness in the deterministic tractography. In Table1, we describe some of them [6, 13]. In the columns we present, respectively, the parameters required by each method, the direction of the starting point, the point $x_{n}$, and its direction $v_{n}$. In these algorithms, $\varepsilon_{n}$ is an independent standard normal random vector, $r_{n}$ is a random vector uniformly distributed over a unit sphere, $\mu$ is the step size, and $\sigma$ is the intensity of artificial noise that is added to generate stochastic tracks.

\section{Entropy-based Method}

In this section, a new Monte Carlo-based fiber tracking algorithm is presented. The main characteristic of this method is that the degree of randomness introduced in the track direction computation and the step size depend on the Shannon entropy of the eigenvalues of the diffusion tensor.

The Shannon entropy $H(X)$ of a discrete random variable $X$ with values in the set $\mathcal{X}=\left\{x_{1}, x_{2}, \ldots, x_{n}\right\}$ is defined as

$$
H(X)=-\sum_{i=1}^{n} p_{i} \ln p_{i}
$$

where $n=|\mathcal{X}|, p_{i}=\operatorname{Pr}\left[X=x_{i}\right]$. The logarithms are taken in base $e$ and entropy is expressed in nats. The Shannon entropy $H(X)$, also denoted by $H(p)$, measures the average uncertainty of random variable $X$ [5].
The main steps of our approach are the following. First, randomness is added to previous $\overline{v_{n-1}}$ using the Shannon entropy (1) of the eigenvalues, which expresses the degree of uncertainty of the diffusion tensor. Second, the resulting vector $\overline{v_{n}}$ is multiplied by the diffusion tensor and, hence, the tensor deflects the perturbed propagation direction towards the major eigenvector direction while limiting the curvature of the deflection, resulting in smoother tract reconstruction [10]. Third, the step size $\mu_{n}$ is small if the uncertainty is large, and vice versa.

Next, we give the details of this approach. From the starting point $x_{0}$, the next positions $x_{n}$ are computed in the following way:

$$
x_{n}=x_{n-1}+\mu_{n-1} \overline{v_{n}} .
$$

The direction $v_{n}$ is given by

$$
v_{n}=D\left(x_{n-1}\right)\left(\overline{v_{n-1}}+\delta_{n-1} r_{n}\right)
$$

and the initial direction $v_{0}$ is taken as

$$
v_{0}=e\left(D\left(x_{0}\right)\right) \text {. }
$$

A random vector $\delta_{n-1} r_{n}$ is computed using

$$
\delta_{n-1}=c \overline{H\left(x_{n-1}\right)}
$$

where $c$ is a constant and $\overline{H\left(x_{n-1}\right)}$ is the normalized Shannon entropy of the eigenvalues, which is given by

$$
\overline{H\left(x_{n}\right)}=\frac{H\left(x_{n}\right)}{\ln 3},
$$

where

$$
H\left(x_{n}\right)=-\sum_{i=1}^{3} \rho_{i}\left(x_{n}\right) \ln \rho_{i}\left(x_{n}\right)
$$

is the Shannon entropy of the normalized eigenvalues:

$$
\rho_{i}\left(x_{n}\right)=\frac{\lambda_{i_{n}}}{\lambda_{1_{n}}+\lambda_{2_{n}}+\lambda_{3_{n}}} .
$$

The maximum value of the Shannon entropy is $\ln 3$ and is obtained when the eigenvalues are equal. In this case, we have the maximum uncertainty in the path direction. On the contrary, the entropy is minimum when two eigenvalues are zero. In this case, there is no uncertainty in the track direction. In quantum mechanics, the entropy of the eigenvalues of the density matrix is used to obtain the Von Neumann entropy of this density matrix [12].

Finally, the step size $\mu_{n}$ is given by

$$
\mu_{n}=1-\overline{H(x)}
$$

Observe that the maximum step size will be obtained with the minimum entropy, i.e., the minimum uncertainty in the path direction. Therefore, an uncertainty increase will provoke a step size decrease. 


\begin{tabular}{|l|c|c|c|c|}
\hline Method & Parameters & $v_{0}$ & $x_{n}$ & $v_{n}$ \\
\hline \hline Deterministic [4] & $\langle\mu>$ & $\overline{\overline{e\left(D\left(x_{0}\right)\right)}}$ & $x_{n-1}+v_{n-1} \mu$ & $\overline{e\left(D\left(x_{n}\right)\right)}$ \\
\hline Hagmann [6] & $\langle\mu, \sigma, k>$ & $\overline{e\left(D\left(x_{0}\right)\right)}$ & $x_{n-1}+v_{n-1} \mu$ & $\overline{\sigma d_{n}+v_{n-1}},\left(v_{n-1} \cdot v_{n}\right)>0$ \\
\hline Prigarin E [13] & $<\mu, \sigma>$ & $\overline{e\left(D\left(x_{0}\right)\right)}$ & $x_{n-1}+v_{n-1} \sigma+\sqrt{\mu} \sigma \varepsilon_{n}$ & $\overline{e\left(D\left(x_{n}\right)\right)},\left(v_{n-1} \cdot v_{n}\right)>0$ \\
\hline Prigarin T1 [13] & $<\mu, \sigma, k>$ & $\overline{e\left(D\left(x_{0}\right)\right)}$ & $x_{n-1}+\mu v_{n-1}+\sqrt{\mu} \sigma \varepsilon_{n}$ & $\overline{D\left(x_{n}\right)^{k} v_{n-1}}$ \\
\hline Prigarin T2 [13] & $<\mu, \sigma, k, c_{0}, c_{1}>$ & $c_{0} \overline{e\left(D\left(x_{0}\right)\right)} ;$ & $x_{n-1}+v_{n} \mu$ & $v_{n-1}+\mu \overline{D\left(x_{n-1}\right)^{k} v_{n-1}}$ \\
& & $a_{0}=0$ & & $-c_{1} v_{n-1}+\sqrt{\mu} \sigma \varepsilon_{n}$ \\
\hline
\end{tabular}

Table 1. $D(x)$ is the diffusion matrix at $x, e$ is the principal eigenvector, $r_{n}$ is a random vector uniformly distributed over a unit sphere, $\mu$ is the step size, $\varepsilon_{n}$ is an independent standard normal random vector and $\sigma$ is the intensity of artificial noise

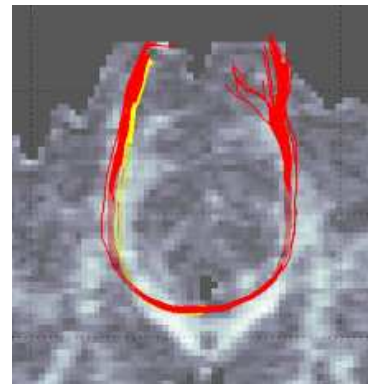

$\mathrm{c}=0.1$

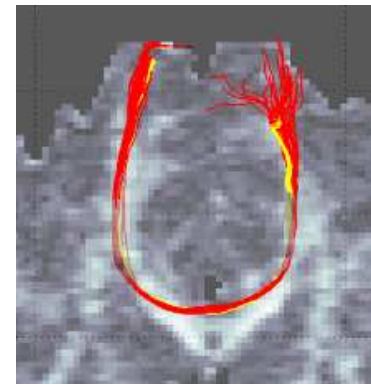

$\mathrm{c}=0.2$

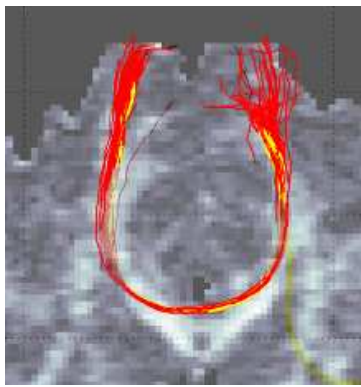

$\mathrm{c}=0.3$

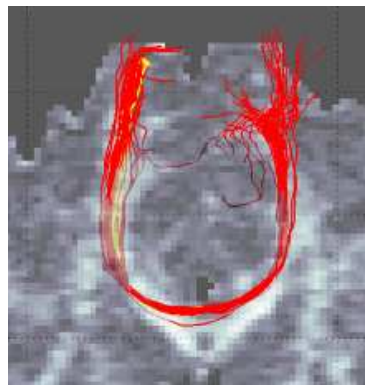

$\mathrm{c}=0.4$

Figure 1. Results obtained with the proposed fiber tracking methods taking a seed in the middle of the genus of the corpus callosum and considering different $c$ values. The yellow track has been obtained by a deterministic approach.

\section{Results}

For the tests we use the Brain dataset courtesy of Gordon Kindlmann at the Scientific Computing and Imaging Institute, University of Utah, and Andrew Alexander, W. M. Keck Laboratory for Functional Brain Imaging and Behavior, University of Wisconsin-Madison.

To properly adjust the parameter $c$ of our method, we place seeds in different well-known anatomical structures of the brain and then we apply the method with $c$ ranging from 0.1 to 0.4 . Obtained results were evaluated by a physician to determine the best one. In all the tests the selected track corresponds to values ranging from 0.2 to 0.3 . In Figure 1, the results obtained when a seed is placed in the genus of the corpus callosum and with $c$ values from 0.1 to 0.4 are shown. Obtained tracks are compared with the deterministic track (in yellow). Observe how the dispersion of the fiber paths increases with $c$.

Our approach with $c=0.2$ is compared with the deterministic method and stochastic T1 and T2 methods. We also compare our method with the tensor deflection technique [10] which uses the entire tensor to determine the di- rection of tract propagation, instead of just the major eigenvector direction. This approach has been used in our tests because it is less sensitive to image noise. For these tests, we place seeds in three known white matter structures identified in a number of studies [15] and that can be reconstructed with fiber tracking techniques. These structures are the fornix, the internal capsule and the genu of the corpus callosum, all represented in Figure 2. In Figure 3, we present some of the obtained results. From top to down, the different images correspond to the proposed approach, the deterministic method, T1 method (with $k=2, \sigma=0.1$, $\mu=0.1$ ), T2 method (with $c_{0}=1, c_{1}=2, k=1, \sigma=0.1$, $\mu=0.1$ ) and the tensor deflection technique. In all the cases, the tracking stops when Fractional Anisotropy (FA) become less than 0.2 [11]. The difference between the compared methods when reconstructing the genu of the corpus callosum is minimal. In all the cases, the anatomical structure is properly tracked. In the fornix case, the proposed method and $\mathrm{T} 2$ perform better than the other ones. Observe that the fornix is perfectly covered by both methods, while the T1 and deterministic approaches generate several erratic fibers. The same behavior can be observed when 

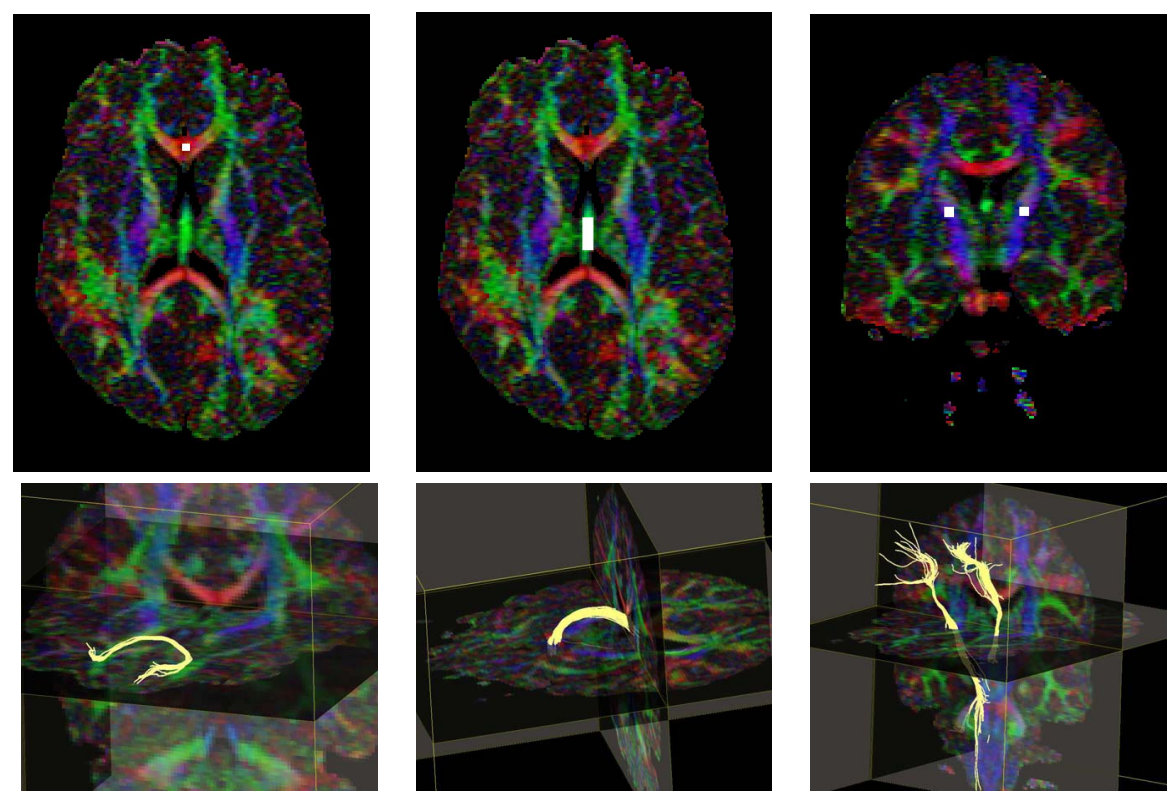

Figure 2. The first row corresponds to seeding regions overlaid over FA maps, genus of corpus callosum, fornix and internal capsule. The second row illustrates the trajectories generated by our method for each seeding region visualized using DTIStudio [7].

tracking the internal capsule. Note that with $\mathrm{T} 2$ and our method the internal capsule is correctly tracked. Although a large experiment with more data sets is necessary to confirm these results, from our experiments we observe that the proposed approach performs better than deterministic and T1 approaches. Moreover, once the $c$ value is set, no more adjustments are required in our method, while in the other stochastic approaches choosing appropriate values for the parameters can be difficult.

\section{Acknowledgements}

We thank Dr. Alberto Prats-Galino, Dpt. Human Anatomy and Embryology, Faculty of Medicine, University of Barcelona for useful discussions regarding the brain anatomy. This project has been funded in part with grant numbers TIN2004-08065-C02-02, TIN2004-07451C03-01 and 2001-SGR-00296.

\section{References}

[1] A. Alexander, K. Hasan, M. Lazar, J. Tsuruda, and D. Parker. Analysis of partial volume effects in diffusiontensor mri. Magnetic Resonance, (45):770-7804, 2001.
[2] P. Basser, J. Matiello, and D. L. Bihan. Estimation of the effective selfdiffusion tensor from the nmr spin echo. Magnetic Resonance, (7):247-254, 1994.

[3] M. Björnemo, A. Brun, R. Kikinis, and C.-F. Westin. Regularized stochastic white matter tractography using diffusion tensor MRI. In Fifth International Conference on Medical Image Computing and Computer-Assisted Intervention (MICCAI'02), pages 435-442, Tokyo, Japan, 2002.

[4] T. E. Conturo, N. F. Lori, T. S. Cull, E. Akbudak, A. Z. Snyder, J. S. Shimony, R. C. McKinstry, H. Burton, and M. E. Raichle. Tracking neuronal fiber pathways in the living human brain. Proceedings National Academy Sciences - Neurobiology, 96:10422-10427, 1999.

[5] T. M. Cover and J. A. Thomas. Elements of Information Theory. John Wiley and Sons, Inc., 1991.

[6] P. Hagmann, J. P. Thiran, L. Jonasson, P. Vandergheynst, S. Clarke, P. Maeder, and R. Meuli. Dti mapping of human brain connectivity: Statistical fibre tracking and virtual dissection. NeuroImage, 19(3):545-554, July 2003.

[7] H. Jiang, P. V. Zijl, J. Kim, G. D. Pearlson, and S. Mori. Dtistudio: Resource program for diffusion tensor computation and fiber bundle tracking. Computer Methods and Programs in Biomedicine, 81:106-116, February 2006.

[8] M. A. Koch, D. G. Norris, and M. Hund-Georgiadis. An investigation of functional and anatomical connectivity using magnetic resonance imaging. NeuroImage, 16(1):241-250, May 2002. 

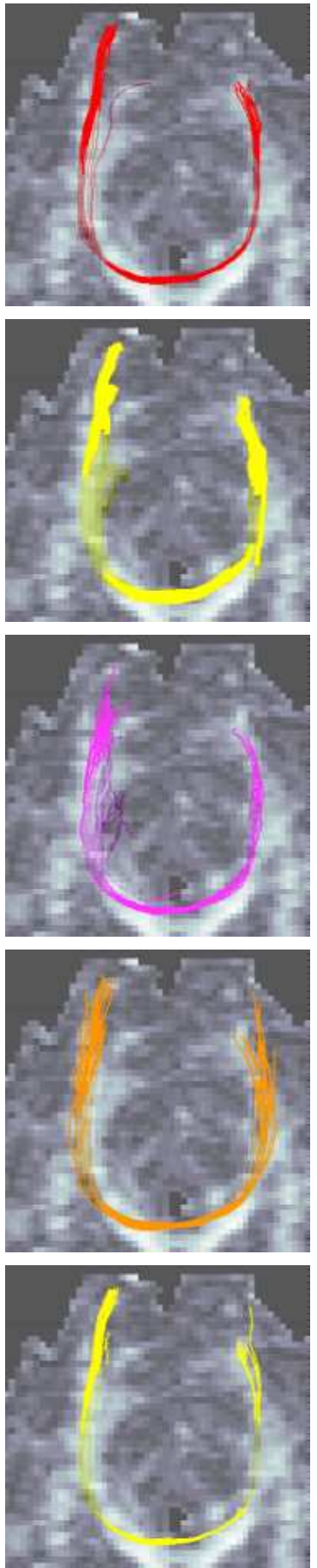
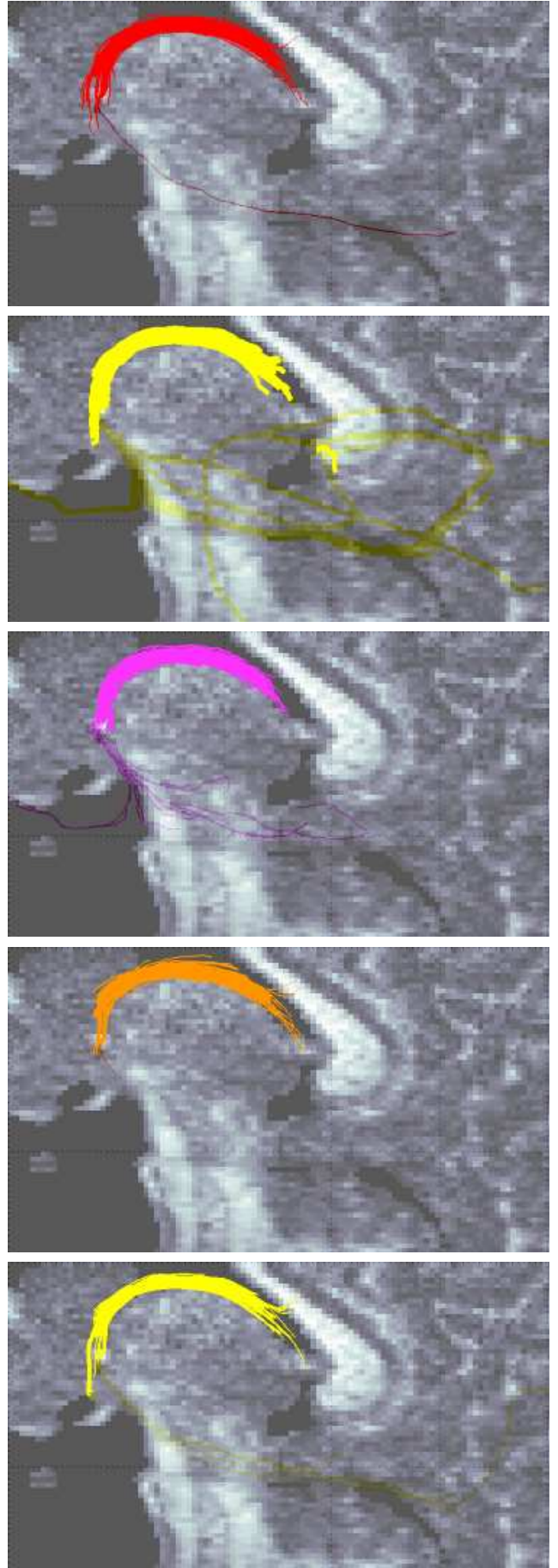
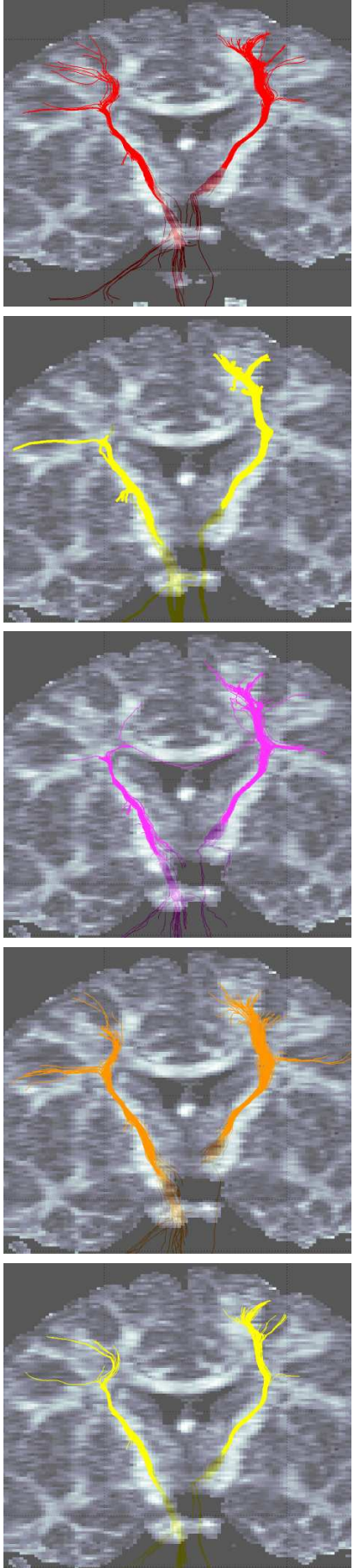

Figure 3. From left to right, each column represents fibers of the genus of corpus callosum, the fornix and the internal capsule, reconstructed by the different methods. From top to down, the compared methods are: the proposed approach, the deterministic method, T1 and T2 methods, and the deflection tensor technique. 
[9] M. Lazar. White Matter Tractography: An Error Analysis and Human Brain Fiber Tract Reconstruction Study. PhD thesis, University of Utah, USA, 2003.

[10] M. Lazar, D. M. Weinstein, J. S. Tsuruda, K. M. Hasan, K. Arfanakis, M. E. Meyerand, B. Badie, H. A. Rowley, V. Haughton, A. Field, and A. L. Alexander. White matter tractography using diffusion tensor deflection. Human Brain Mapping, (18):306-321, 2003.

[11] S. Mori and P. C. M. van Zijl. Fiber tracking: principles and strategies - a technical review. NMR in Biomedicine, (15):468-480, 2002.

[12] A. Peres. Quantum Theory: Concepts and Methods. Kluwer Academic Publisher, 1993.

[13] S. M. Prigarin and K. Hahn. Stochastic algorithms for white matter fiber tracking and the inference of brain connectivity from $\mathrm{mr}$ diffusion tensor data, December 2004.

[14] D. Tuch, T. Reese, M.Wiegell, N.Makris, J.Belliveau, and V.Wedeen. High angular resolution diffusion imaging reveals intravoxel white matter fiber heterogeneity. Magnetic Resonance in Medicine, (48):577-582, 2002.

[15] S. Wakana, H. Jiang, L. M. Nagae-Poetscher, P. C. M. van Zijl, and S. Mori. Fiber tract-based atlas of human white matter anatomy. Radiology, 1(230):77-87, 2004.

[16] C.-F. Westin, S. Peled, H. Gudbjartsson, R. Kikinis, and F. A. Jolesz. Geometrical diffusion measures for MRI from tensor basis analysis. In ISMRM '97, page 1742, Vancouver Canada, April 1997. 